\title{
HD 17156b: a transiting planet with a 21.2-day period and an eccentric orbit
}

\author{
M. Barbieri ${ }^{1}$, R. Alonso ${ }^{1}$, G. Laughlin ${ }^{2}$, J. M. Almenara ${ }^{3}$, R. Bissinger ${ }^{4}$, D. Davies ${ }^{5}$, \\ D. Gasparri ${ }^{6}$, E. Guido ${ }^{7}$, C. Lopresti ${ }^{8}$, F. Manzini ${ }^{9}$, and G. Sostero ${ }^{7}$ \\ 1 LAM, Traverse du Siphon, BP 8, Les Trois Lucs, 13376 Marseille Cedex 12, France \\ e-mail: [mauro.barbieri;roi.alonso]@oamp.fr \\ 2 University of California Observatories, University of California at Santa Cruz Santa Cruz, CA 95064, USA \\ e-mail: laugh@ucolick.org \\ Instituto de Astrofísica de Canarias, C/Vía Láctea s/n, 38200 La Laguna, Spain \\ 4 Racoon Run Observatory, Pleasanton, CA, USA \\ 23819 Ladeene Avenue, Torrance, CA 90505, USA \\ Università di Bologna, Dipartimento di Astronomia, via Ranzani 1, 40127 Bologna, Italy \\ 7 Associazione Friulana di Astronomia e Meteorologia, Piazza Miani nr. 2, 33047 Remanzacco, Italy \\ 8 Istituto Spezzino Ricerche Astronomiche, via Castellazzo 8/d, 19123 La Spezia, Italy \\ 9 Stazione Astronomica di Sozzago, 28060 Sozzago, Italy
}

Received 4 October 2007 / Accepted 24 October 2007

ABSTRACT

\begin{abstract}
We report the detection of transits by the $3.1 M_{\text {Jup }}$ companion to the $V=8.17$ G0V star HD 17156 . The transit was observed by three independant observers on Sep. 9/10, 2007 (two in central Italy and one in the Canary Islands), who obtained detections at confidence levels of $3.0 \sigma, 5.3 \sigma$, and $7.9 \sigma$, respectively. The observations were carried out under the auspices of the Transitsearch.org network, which organizes follow-up photometric transit searches of known planet-bearing stars during the time intervals when transits might be expected to occur. Analyses of the $7.9 \sigma$ data set indicates a transit depth $d=0.0062 \pm 0.0004$ and a transit duration $t=186 \pm$ $5 \mathrm{~min}$. These values are consistent with the transit of a Jupiter-sized planet with an impact parameter $b=a \cos i / R_{\star} \sim 0.8$. This planet occupies a unique regime among known transiting extrasolar planets, both as a result of its large orbital eccentricity $(e=0.67)$ and long orbital period $(P=21.2 \mathrm{~d})$. The planet receives a 26 -fold variation in insolation during the course of its orbit, which will make it a useful object for characterizing exoplanetary atmospheric dynamics.
\end{abstract}

Key words. binaries: eclipsing - planetary systems - stars: individual: HD 17156 - techniques: photometric

\section{Introduction}

During the past several years, the discovery rate of transiting planets has begun to increase rapidly, to the point where twenty transiting planets with secure characterizations are currently known ${ }^{1}$. This aggregate consists mostly of short-period hot-Jupiter type planets, with prototypical examples being HD 209458b (Charbonneau et al. 2000; Henry et al. 2000) and HD 189733b (Bouchy et al. 2005). These planets tend to have $M \sim 1 M_{\text {Jup }}, 2 \mathrm{~d}<P<5 \mathrm{~d}$, and tidally circularized orbits.

In the past years, two remarkable discoveries have significantly extended the parameter space occupied by known transiting planets. HD 147506b (Bakos et al. 2007) with $M=$ $8.04 M_{\text {Jup }}$ is by far the most massive planet known to exhibit transits. It also has the longest orbital period (5.63 days) and a startlingly large orbital eccentricity, $e \sim 0.5$. At the other end of the mass scale, Gl 436b (Butler et al. 2004; Gillon et al. 2007) has $M=0.07 M_{\mathrm{Jup}}$, a 2.64-day orbital period, and an eccentricity $e=0.15 \pm 0.01$ (Deming et al. 2007). These two planets differ in mass by more than a factor of one hundred, and their significant non-zero eccentricities are also capable of imparting important information.

\footnotetext{
${ }^{1}$ Extrasolar Planets Encyclopedia http://exoplanet. eu
}

At present, the infrared observations of transiting extrasolar planets by Spitzer present an incomplete and somewhat contradictory overall picture. It is not understood how the wind vectors and temperature distributions on the observed planets behave as a function of pressure depth and of planetary longitude and latitude. Most important, the effective radiative time constant in the atmospheres of short-period planets remains unmeasured; as a result, dynamical calculations of the expected planetwide flow patterns (Cho et al. 2003; Cooper \& Showman 2005; Burkert et al. 2005; Langton \& Laughlin 2007; Dobbs-Dixon \& Lin 2007) have come to no consensus regarding how the surface flow should appear. This lack of agreement between the models stems in large part from the paucity of unambiguous measurements of the radiative time constant in the atmosphere. What is needed is a transiting planet with both a long-period orbit and a large orbital eccentricity. If such a planet were known, then one could use Spitzer to obtain infrared time-series photometry of the planet during the periastron passage. The transit guarantees knowledge of both the geometric phase function and the planetary mass. This information would in turn allow a measured rate of increase in flux to inform us of the planet's atmospheric radiative time constant in the observed wavelength regime.

The orbital periods of the known transiting planets are all significantly shorter than 6 days. This bias is due both to the 
intrinsically lower geometric probability of transit as one moves to longer periods and to the fact that ground-based wide-field transit surveys that rely on photometry folding becoming very significantly incomplete for planets with orbital periods longer than 5 days. If one wants to detect longer-period transiting planets from the ground, a more productive strategy is to monitor known RV-detected, planet-bearing stars at the times when the radial velocity solution suggests that transits may occur. This strategy has the further advantage of producing transits around stars that tend to be both bright and well-suited to follow-up observations.

Long-period transiting planets present an ideal observing opportunity for small telescope observers. Seagroves et al. (2003) suggest that a global network of telescopes, all capable of $\sim 1 \%$ photometry can easily outperform a single large telescope in terms of the efficiency of transit recovery. Since its inception in 2002, the Transitsearch. org network has conducted followup searches of a number of intermediate-period planets (see e.g. Shankland et al. 2006).

The Doppler-based discovery of HD $17156 \mathrm{~b}$ has recently benn published by the N2K consortium (Fischer et al. 2007). The planet has $M \sin i=3.12 M_{\text {Jup }}$, with $P=21.22$ days and $e \sim 0.67$. Fischer et al. (2007) report that the $V=8.17 \mathrm{G0V}$ host star has $M=1.2 M_{\odot}$ and $R=1.47 R_{\odot}$. The planet's semimajor axis $a=0.15 \mathrm{AU}$ thus indicates a periastron distance of $a_{\text {min }}=0.0495 \mathrm{AU}=7.2 R_{\star}$. A best fit to the radial velocities indicates longitude a of periastron $\omega=121 \pm 11^{\circ}$. The orbital orientation is favorable, yielding an a-priori geometric transit probability of $P \sim 13 \%$.

In their discovery paper, Fischer et al. (2007) report 241 individual photometric measurements obtained over a 179-day interval, with a mean dispersion $\sigma=0.0024 \mathrm{mag}$. No significant rotation-induced periodicity was seen. Together, the observations sampled approximately $25 \%$ of the $1-\sigma$ transit window, and no evidence of a transit was observed. After the Fischer et al. (2007) discovery paper was made public, the star was added to the Transitsearch.org candidates list $^{2}$ and observers throughout the Northern Hemisphere have been repeatedly encouraged to obtain photometry of the $\operatorname{star}^{3}$. The first available window of opportunity occurred on 9/10 Sep., 2007, with the transit midpoint predicted to occur at HJD $2454353.65 \pm 0.30$.

\section{Observations}

We collected data from different observatories during the night of September 9/10, 2007. The following instrumentation was used:

- Almenara: observations were gathered in the $R$ band and $7 \mathrm{~s}$ of exposure time with the TELAST $0.30 \mathrm{~m}$ telescope, a stellar photometer devoted to IAC Asteroseismology programs. The telescope is an $f / 10$ Schmidt-Cassegrain catadioptric, and an SBIG STL-1001E CCD camera provides a field of view of $29^{\prime} \times 29^{\prime}\left(\right.$ scale $\left.1.7^{\prime \prime} / \mathrm{px}\right)$. The night was windy, affecting the telescope (totally exposed), appearing to double and even triple the stars. The gap in the center of the observations was caused by the loss of the guide star due to the wind.

- Bissinger: observations were made from Pleasanton, California USA using a $0.4 \mathrm{~m}$ diameter modified SchmidtCassegrain telescope operating at $f / 6$ with an SBIG

\footnotetext{
${ }^{2}$ http://207.111.201.70/transitsearch/dynamiccontent/ candidates.html

${ }^{3}$ See www. oklo.org
}

ST-10XME CCD camera and a Bessell $I$ band filter. Imaging began at 04:05 UT on Sep. 10 and ended at 09:09 UT on Sep. 10 with an exposure cadence of 43 s. Bins of 15 exposures were made producing a flat light curve with an rms of $0.003 \mathrm{mag}$.

- Gasparri: the telescope used is a commercial $0.25 \mathrm{~m} \mathrm{f} / 4.8$ Newtonian, located near Perugia, Italy. The camera is an SBIG ST-7XME with a KAF-0402 CCD providing a field of view of $19.8^{\prime} \times 13.2^{\prime}$ with sampling of $1.55^{\prime \prime} / \mathrm{px}$. The photometric observation started at 20 UT on Sep. 9 and stopped at 04 UT on Sep. 10. The presence of some clouds and veils limited the useful data to 00-02 UT of 10 Sep. Exposures were made through a near-IR filter and are of $20 \mathrm{~s}$ duration, with 339 useful images collected.

- Lopresti: observations were conducted at La Spezia, Italy with a Maksutov-Newton telescope of $0.18 \mathrm{~m}$ diameter $\mathrm{f} / 4$ and an SBIG ST-10xme CCD camera, with a framed field of $70^{\prime} \times 47^{\prime}$ (scale $\left.1.7^{\prime \prime} / \mathrm{px}\right)$. Observations ran from $22 \mathrm{UT}$ Sep. 9 through 04 UT Sep. 10. Exposure times were 5 s. A total of $580 R$ band images were collected.

- Manzini: the Stazione Astronomica di Sozzago is an observatory located in Sozzago (Novara) Italy (international code IAU A12). Observations were conducted with a $0.40 \mathrm{~m}$ Cassegrain telescope $f / 6.7$, equipped with a CCD camera Hi-SIS43ME $\left(\right.$ FoV $18^{\prime} \times 11^{\prime}$, scale $\left.0.7^{\prime \prime} / \mathrm{px}\right)$. Useful data were collected until 20 UT Sep. 9, when clouds intervened.

\section{Data analysis}

All the raw images were calibrated in the standard way: each observer took a series of images to correct for the irregular pixel sensivity (flat-fielding) and dark current effects. Out of the 6 datasets, only three cover the central transit window. These were obtained by the amateur astronomers Lopresti and Gasparri (in Italy) and by Almenara (at the IAC). Unfortunately, the data are irregular in their coverage of the transit and in their precision.

The three data sets were analyzed with IDL routines to perform simple aperture photometry. The center of the aperture was calculated by a Gaussian fit, and the aperture was held fixed (to 15-20 px in radius, depending on the data set). We removed the sky background contribution after estimating of its value in an annulus around the target aperture. The brightest stars in the field were measured in the same way, and a reference light curve was constructed by adding the flux of these stars. The target flux was divided by this reference to get the final normalized curve. The data included in this detailed analysis are:

- Almenara (A): from $6 \mathrm{~h}$ before center to $3.4 \mathrm{~h}$ after transit center. The dispersion is high at the beginning and better at the end, decreasing from $0.5 \%$ to $0.4 \%$ (in a k-sigma filtered version of the original light curve). Two stars were used to build the reference light curve.

- Gasparri (G): from $2 \mathrm{~h}$ before center to $1.1 \mathrm{~h}$ after center. Three stars were used to build the reference star. The rms of the residuals is $\sim 0.4 \%$. Soon before transit center there was a rise in the light curve that we were not able to correct, due to the clouds and veils affecting the target differently and each of the stars used to build the reference.

- Lopresti (L): from $4 \mathrm{~h}$ before transit center to $1.5 \mathrm{~h}$ after transit center. The last $45 \mathrm{~min}$ were taken with a $180^{\circ}$ rotation of the CCD (due to the mount configuration). We have not been able to fully correct for the effect of this rotation, as data are sensitive to uncorrected minor flat field effects. To avoid the introduction of offsets, we have thus ignored these 


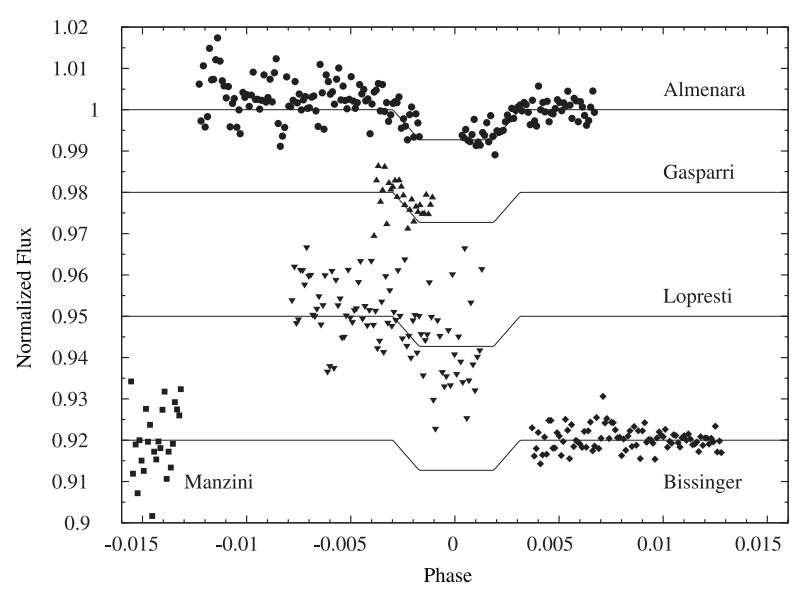

Fig. 1. Normalized light curves of HD 17156 at the moment of transit and the best-fitted trapezoid to the Almenara data set (solid lines). Data sets of different observers have been shifted relative each other for clarity. The transit is centered at HJD 2454353.61

last data. Three stars were used to build the reference light curve. The rms of the residuals is $\sim 0.9 \%$.

The final data sets are plotted in Fig. 1. We performed a fit to a trapezoidal function, with four free parameters: center, width, depth, and size of the transition between the two levels of the trapezoid. The significance of the transit detection was then evaluated as the value of the depth of the trapezoid divided by the dispersion of the fit. Thus, we obtain a $5.6 \sigma$ detection for the (A) set. This value increases to $7.9 \sigma$ once the baseline is corrected by a parabolic fit to the parts of the light curve outside of the trapezoid, plus an extra margin of 0.001 in phase to avoid the inclusion of points inside transit. For the (L) and (G) data sets, the free parameters for the trapezoid fitting were only the depth and center. The other two parameters were set to the result of the fit in the (A) set. We obtain the values of $5.3 \sigma$ and $2.97 \sigma$, respectively.

Due to the lack of quality of the three data sets, we believe it is too dangerous to perform a combined analysis; in particular the zero offsets are not too clear in the Gasparri (G) and Lopresti (L) data sets, and they might dominate the result of a fitting to a combined light curve. We thus analyzed the most homogeneous and least noisy part of the data, namely the egress recorded in the (A) set, to determine the main characteristics of the transit. We employed two strategies: fitting to a trapezoid to estimate the depth of the transit, and fitting to a model of an eccentric transit following the formalism of Giménez (2006).

To correct for the baseline, the trapezoid fit to the egress was performed in two steps: (1) a first trapezoid is fitted, removed from the light curve, and a line is fitted to the residuals. This line is removed from the original light curve and (2) a second trapezoid is fitted, providing the values of the depth and time of egress. The errors are evaluated by a bootstrap analysis, performing 20000 tests with data sets in which $50 \%$ of the residual points were randomly re-sorted, and the best-fitted model was re-added to the residuals to build the data set. The same two-step fitting was performed in each data set. The depth of the trapezoid was found to be $0.0062 \pm 0.0004$.

The second strategy consisted in a fit to the equations of Giménez (2006). The fitted parameters were the phase of start of the transit, $k, i$, and three coefficients defining a parabolic baseline correction. The two non-linear limb-darkening coefficients were fixed to $u_{+}=0.65$ and $u_{-}=-0.05$ (see Giménez 2006,
Table 1. Transit fit and planetary parameters for HD $17156 \mathrm{~b}$.

\begin{tabular}{lr}
\hline \hline Parameter & Value \\
\hline$T_{\text {mid }}(\mathrm{HJD})$ & $2454353.61 \pm 0.02$ \\
$\phi_{\text {egress }}$ & $0.003050 \pm 0.000075$ \\
Transit duration (day) & $0.1294 \pm 0.0367$ \\
$k=R_{\mathrm{p}} / R_{\star}$ & $0.08007 \pm 0.0028$ \\
$i(\mathrm{deg})$ & $87.89 \pm 0.10$ \\
\hline$R_{\mathrm{p}}\left(R_{\mathrm{Jup}}\right)$ & $1.15 \pm 0.11$ \\
$\rho_{\mathrm{p}}\left(\mathrm{g} / \mathrm{cm}^{3}\right)$ & $2.58 \pm 0.84$ \\
\hline
\end{tabular}

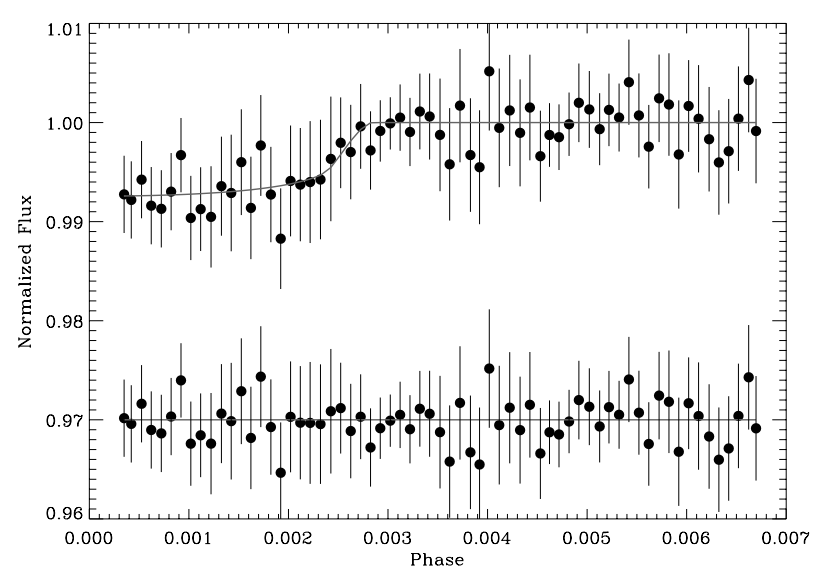

Fig. 2. Top: normalized phase plot of the egress of the transit of HD 17156 from the Almenara data set. The error bar in each point it was calculated as the standard deviation of the 20 points closest to point $i$. The plotted error bars are 2 times this quantity. The overplotted line is the best-fitted model using the formalism of Gimenez as described in the text. Bottom: the residuals of the fit.

for a definition of these coefficients) from the tables of Claret (2000) for ATLAS stellar models. The eccentricity and the longitude of the periastron were fixed to values obtained from Fischer et al. (2007). The best solution was obtained by minimizing the $\chi^{2}$ between the model and the observations using the algorithm AMOEBA (Press et al. 1992). The errors were estimated by a bootstrap analysis similar to the one described above, performing 1500 tests. The best-fitted values using this technique are reported in Table 1.

Based on the inclination, the planet mass is then $M_{\mathrm{p}}=3.12 \pm$ $0.5 M_{\text {Jup }}$. Based on the radius of the star and the $k$ determination, the radius of the planet is $R_{\mathrm{p}}=1.15 \pm 0.11 R_{\mathrm{Jup}}$, and the resulting planet mean density is $\rho_{\mathrm{p}}=2.58 \pm 0.84 \mathrm{~g} / \mathrm{cm}^{3}$. These properties are summarized in Table 1, the final fit and the residuals are plotted in Fig. 2.

As an additional test, we checked the Hipparcos photometry (Perryman \& ESA 1997). Hipparcos observed HD 17156 (HIP 13192) on 142 occasions with a standard deviation of $0.0013 \mathrm{mag}$. An inspection of the light curve folded with the orbital period of the planet shows only two photometric points close to the transit window.

During the night of Sep. 30/Oct. 1, HD 17156b was observed from the Mount Laguna Observatory in southern California. The team composed of William Welsh, Abhijith Rajan, Jonathan Irwin, Philip Nutzman, and David Charbonneau kindly report to us (Charbonneau, personal communication) that the transit ingress was observed near Oct. 1 UT 6:30, and a flat-bottomed event followed, egress was lost due to clouds.

In addition, observer Davies, located in Torrance, California, obtained $\sim 10000$ CCD images which cover the full transit window. A preliminary analysis confirms the detection of the transit, 
and a more detailed analysis will be presented in a forthcoming paper (Irwin et al., in preparation).

\section{Discussion}

The detection of transits by a planet with a three-week orbital period demonstrates the utility of ad hoc networks of small telescopes for obtaining photometric follow-up of planets whose orbital parameters have been determined via Doppler radial velocities. Indeed, the transits of HD $17156 \mathrm{~b}$ offer a plethora of interesting opportunities for follow-up observations.

With its high orbital eccentricity and small periastron distance, HD 17156b appears to bear a curious kinship to HD 80606b, HD 147506b, and HD 108147b. All three of these planets occupy a locus of the $a-e$ plane where they should be actively undergoing tidal dissipation; therefore they should be generating significant quantities of excess interior heat. Our measurement indicates that tidal heating is not significantly inflating the planetary radius. The nominal $R=1.1 R_{\text {Jup }}$ radius predicted by baseline models (e.g. those of Bodenheimer et al. 2003) is confirmed by our observations.

Follow-up photometric measurements during future transits will allow a more accurate determination of the orbital inclination of HD 17156b. An improved value for $i$, in turn, will generate an accurate assessment of the likelihood that the planet can be observed by Spitzer in secondary transit and will enable a much-improved constraint on the still-uncertain radius of the parent star. In the event that secondary transits can be observed, a direct measurement of the excess tidally-generated luminosity from the planet is a distinct possibility (see e.g. Deming et al. 2007).

As a consequence of its highly eccentric orbit, HD 17156b experiences a 26 -fold variation in insolation during the 10.6-day interval between periastron and apoastron. This extreme radiative forcing may drive interesting, and potentially observable, dynamical atmospheric flows on the planet (Langton \& Laughlin 2007). The large tidal forces experienced during periastron have almost certainly forced the planet into pseudo-synchronous rotation (e.g. Goldreich \& Peale 1968; Hut 1981; Papaloizou \& Ivanov 2005). Rotationally induced modulation in the infrared light curve following periastron is potentially observable and may be very useful when selecting between the current divergent predictions for the actual value of the pseudo-synchronous spin frequency.

HD $17156 \mathrm{~b}$ is quite massive, as is often the case for planets orbiting one member of a binary pair (Desidera \& Barbieri 2007), and its eccentricity is large. These characteristics favor a formation scenario involving migration and/or dynamical evolution in the presence of a sufficiently close external perturber. Such a perturber could be either a companion star or an additional planet(s) in the system.

For some of the close-in planets with $m \sin i>1.5 M_{\text {Jup }}$ orbiting single stars, there are already indications of significant dynamical perturbations. For example, HD 118203b, HD 68988b and HIP 14810b all have anomalously large eccentricities that may indicate of additional, perturbing companions, perhaps with masses below (or periods longer than) the threshold of immediate radial velocity detection. This is certainly the case for HD 68988b and HIP 14810b, which both have longperiod planetary companions (Butler et al. 2006). Due to mutual perturbations, the eccentricities of the bodies in the precursor system may have grown to the point where crossing orbits were achieved. Repeated close encounters among the planets would have then generated a period of chaotic evolution that typically terminates with the ejection of one planet on a hyperbolic trajectory (Marzari \& Weidenschilling 2002).

Alternately, a stellar companion could also effectively trigger dynamical evolution or instability in a precursor system, eventually leading to the current configuration (for some examples, see Marzari \& Barbieri 2007a,b; Wu \& Murray 2003). With reference to a stellar companion, a quick inspection of POSSI, POSSII, and 2MASS images do not reveal any clear association between faint field stars and HD 17156. The only potentially interesting source is 2 MASS $02494068+7144583$. It shows an appreciable proper motion, but in the opposite direction of the proper motion of HD 17156. The star lies 22.2" from HD 17156; if they are at the same distance, the apparent separation is $\sim 1740$ AU.

A combination of continued radial velocity monitoring of HD 17156, in conjunction with accurate measurements of successive transit midpoints, gives hope for detecting and accurately characterizing additional bodies in the system via a novel set of constraints.

Acknowledgements. Support for M.B. is provided by Agence Nationale de la Recherche (France). Support for R.A. is provided by the CNES under contract 02/0425. Support for Transitsearch. org was provided by NASA under grant NNG04GN30G through the OSS program. We wish to acknowledge the financial support from Spanish grants ESP2004-03855-C03 and AYA2004-04462. We thank Dr. David Charbonneau for generous advice. M.B. thanks C. Lopresti for useful discussions, and R. Calanca is gratefully aknowledged for his invaluable support in communicating exoplanetary science within the Italian amateur astronomical community.

\section{References}

Bakos, G. A., et al. 2007, [arXiv:0705.0126]

Bodenheimer, P., Laughlin, G., \& Lin, D. N. C. 2003, ApJ, 592, 555

Bouchy, F., Udry, S., Mayor, M., et al. 2005, A\&A, 444, L15

Burkert, A., Lin, D. N. C., Bodenheimer, P. H., Jones, C. A., \& Yorke, H. W. 2005, ApJ, 618, 512

Butler, R. P., Vogt, S. S., Marcy, G. W., et al. 2004, ApJ, 617, 580

Butler, R. P., Wright, J. T., Marcy, G. W., et al. 2006, ApJ, 646, 505

Charbonneau, D., Brown, T. M., Latham, D. W., \& Mayor, M. 2000, ApJ, 529, L45

Cho, J. Y.-K., Menou, K., Hansen, B. M. S., \& Seager, S. 2003, ApJ, 587, L117

Claret, A. 2000, A\&A, 363, 1081

Cooper, C. S., \& Showman, A. P. 2005, ApJ, 629, L45

Deming, D., Harrington, J., Laughlin, G., et al. 2007, [arXiv:0707. 2778]

Desidera, S., \& Barbieri, M. 2007, A\&A, 462, 345

Dobbs-Dixon, I., \& Lin, D. N. C. 2007, [arXiv: 0704 . 3269]

Fischer, D. A., et al. 2007, [arXiv:0704 . 1191]

Gillon, M., Pont, F., Demory, B.-O., et al. 2007, A\&A, 472, L13

Giménez, A. 2006, A\&A, 450, 1231

Goldreich, P., \& Peale, S. J. 1968, ARA\&A, 6, 287

Henry, G. W., Marcy, G. W., Butler, R. P., \& Vogt, S. S. 2000, ApJ, 529, L41

Hut, P. 1981, A\&A, 99, 126

Langton, J., \& Laughlin, G. 2007, ApJ, 657, L113

Marzari, F., \& Weidenschilling, S. J. 2002, Icarus, 156, 570

Marzari, F., \& Barbieri, M. 2007a, A\&A, 467, 347

Marzari, F., \& Barbieri, M. 2007b, A\&A, 472, 643

Papaloizou, J. C. B., \& Ivanov, P. B. 2005, MNRAS, 364, L66

Perryman, M. A. C., \& ESA 1997, ESA Spec Publ, 1200

Press, W. H., Teukolsky, S. A., Vetterling, W. T., \& Flannery, B. P. 1992, Numerical recipes in FORTRAN, The art of scientific computing, 2nd ed., (Cambridge: University Press)

Seagroves, S., et al. 2003, PASP, 115, 1355

Shankland, P. D., Rivera, E. J., Langhlin, G., et al. 2006, ApJ, 653, 700

Wu, Y., \& Murray, N. 2003, ApJ, 589, 605 